\title{
CONVEX LATTICE POLYGONS OF MINIMUM AREA
}

\section{R.J. SIMPSON}

A convex lattice polygon is a polygon whose vertices are points on the integer lattice and whose interior angles are strictly less than $\pi$ radians. We define $a(2 n)$ to be the least possible area of a convex lattice polygon with $2 n$ vertices. A method for constructing convex lattice polygons with area $a(2 n)$ is described, and values of $a(2 n)$ for low $n$ are obtained.

\section{INTRODUCTION AND KNOWN RESULTS}

A convex lattice polygon is a polygon whose vertices are points on the integer lattice and whose interior angles are strictly less than $\pi$ radians. A lattice polygon with $v$ vertices will be called a $v$-gon. In this paper we investigate the function $a(v)$ which gives the least possible area of a convex $v$-gon. A convex $v$-gon with area $a(v)$ is called minimal.

In our first theorem we show that finding $a(v)$ for a given $v$ is equivalent to finding $g(v)$ which is the least possible number of lattice points in the interior of a convex $v$-gon.

THEOREM 1. For $v \geqslant 3$,

$$
a(v)=g(v)+v / 2-1
$$

Proof: Consider a convex $v$-gon with $g(v)$ interior lattice points. Suppose that $A, B$ and $C$ are three vertices with $A$ adjacent to $B, B$ adjacent to $C$, and that the lattice point $X$ lies on the edge joining $A$ and $B$ and is distinct from $A$ and $B$. We can construct a new polygon by replacing the edges $A B, B C$ with $A X, X C$. This new polygon is still convex and contains $g(v)$ interior lattice points. Repeating the process we can obtain a convex $v$-gon with $g(v)$ interior lattice points and whose circumference includes no lattice points other than the vertices. By Pick's Theorem [2] the area of this polygon is

$$
g(v)+v / 2-1
$$

which implies

$$
a(v) \leqslant g(v)+v / 2-1
$$

\footnotetext{
Received 6 November 1989
}

Copyright Clearance Centre, Inc. Serial-fee code: 0004-9729/90 \$A2.00+0.00. 
Now suppose we have a convex $v$-gon with area $a(v)$. The construction described above reduces the area of a $v$-gon so the only lattice points on the circumference of the polygon are its vertices, and hence by Pick's Theorem,

$$
a(v) \geqslant g(v)+v / 2-1 .
$$

Together (1) and (2) give the required result.

REMARK. Since $g(v)$ is always an integer the theorem implies that $a(v)$ is an integer when $v$ is even and an integer plus one half when $v$ is odd.

The function $g(v)$ has been studied extensively by Arkinstall [1] and Rabinowitz $[4,5]$. To apply the methods of this paper it is more convenient to use $a(v)$. In Table 1 we list some results obtained by these authors.

\begin{tabular}{rcc}
$v$ & $a(v)$ & $g(v)$ \\
\hline 3 & 0.5 & 0 \\
4 & 1.0 & 0 \\
5 & 2.5 & 1 \\
6 & 3.0 & 1 \\
7 & 6.5 & 4 \\
8 & 7.0 & 4 \\
9 & 10.5 & 7 \\
10 & 14.0 & 10 \\
11 & {$[15.5,21.5]$} & {$[11,17]$} \\
12 & {$[17.0,24.0]$} & {$[12,19]$} \\
13 & {$[19.5,32.5]$} & {$[14,27]$} \\
14 & {$[21.0,40.0]$} & {$[15,34]$} \\
15 & {$[23.5,54.5]$} & {$[18,48]$} \\
16 & {$[25.0,63.0]$} &
\end{tabular}

Table 1: The square brackets define a closed interval known to contain the value. The results for $v=5$ and $v=6$ are due to Arkinstall [1], those for higher values of $v$ are due to Rabinowitz [4].

The main purpose of this paper is to obtain a sufficient characterisation of minimal $v$-gons for even $v$ to find $a(v)$ with a computer. This characterisation is derived in the next $t$ wo sections. We then use this characterisation to extend the list of known values of $a(v)$. In the last section we consider $v$-gons for odd $v$ and discuss our results. 


\section{Characterisation of a minimal 2 n-gon}

In this and the next section we consider lattice polygons with an even number of vertices, and show that without loss of generality a minimal $2 n$-gon may be assumed to have various properties.

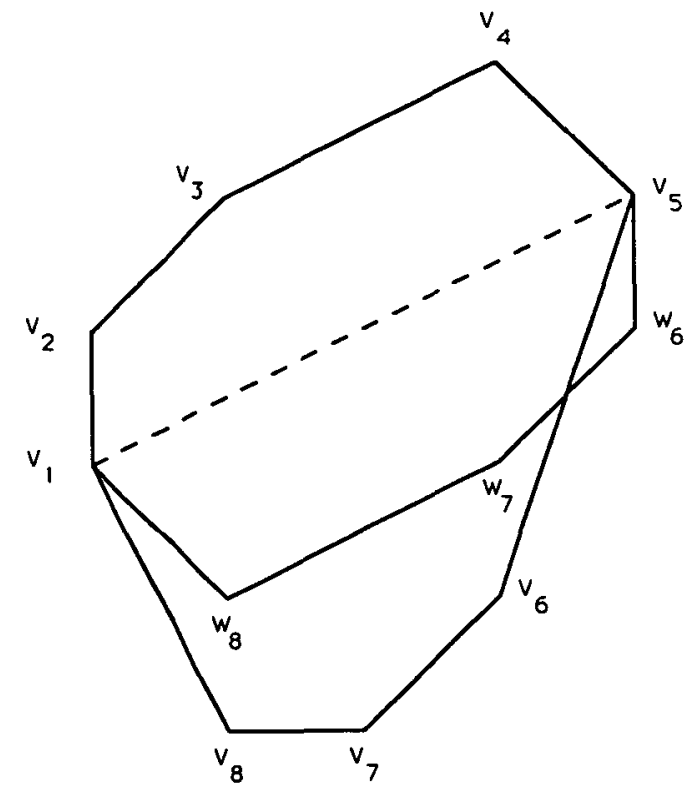

Diagram 1

ThEOREM 2. For every $n$ greater than 1 there exists a parallel-sided minimal 2n-gon.

Proof: Let $K$ be a convex lattice $2 n$-gon with area $a(2 n)$ and vertices $V_{1}, V_{2}, \ldots, V_{2 n}$. For any integer $i \in[1, n]$ the line segment $V_{i} V_{i+n}$ partitions the polygon into two areas, say $A_{1}$ and $A_{2}$. Without loss of generality suppose $A_{1} \leqslant A_{2}$. We can then form a parallel-sided lattice polygon $L$ using vertices $W_{1}, W_{2}, \ldots, W_{2 n}$ where

$$
W_{j}=V_{i-1+j} \text { for } j=1, \ldots, n+1
$$

and for $j=n+2$ to $2 n$ we set $W_{j}$ so that the edge from $W_{j-1}$ to $W_{j}$ is parallel and equal in length to the edge from $W_{j-n-1}$ to $W_{j-n}$. See Diagram 1. This gives a new $2 n$-gon with area $2 A_{1}$ which is at most equal to $a(2 n)$, since $K$ is minimal. If $L$ is convex we must have $2 A_{1}$ equal to $a(2 n)$ and we are done. It remains to show that we can always choose $i$ so that $L$ is convex.

Consider Diagram 2. This shows part of the circumference of $K$ and line segments $V_{1} V_{n+1}$ and $V_{2} V_{n+2}$. The first line segment, which corresponds to taking $i=1$, will 


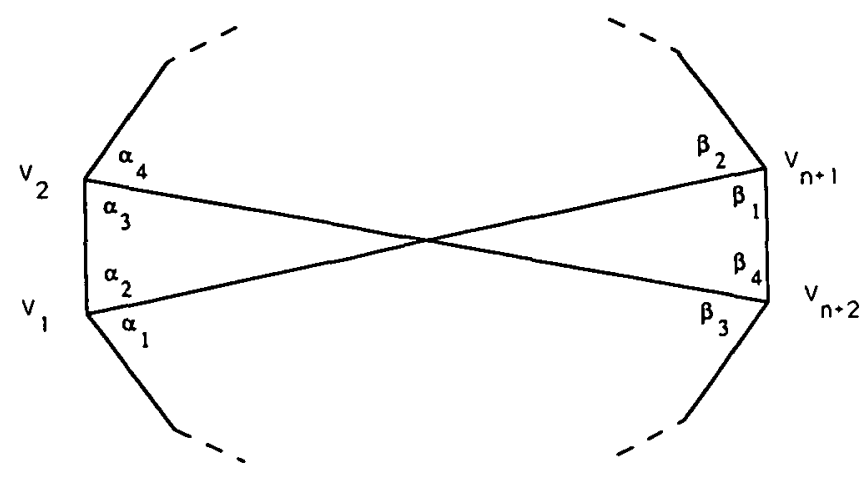

Diagram 2

produce a convex $2 n$-gon $L$ provided neither $\alpha_{1}+\beta_{1}$ nor $\alpha_{2}+\beta_{2}$ is at least $\pi$. If this is so we are done.

We cannot have both these sums being at least $\pi$ since this would imply either $\alpha_{1}+\alpha_{2}$ or $\beta_{1}+\beta_{2}$ is at least $\pi$, contradicting the convexity of $K$. Assume then that

$$
\begin{aligned}
& \alpha_{1}+\beta_{1} \geqslant \pi, \\
& \alpha_{2}+\beta_{2}<\pi .
\end{aligned}
$$

By the convexity of $K$ we have

$$
\text { and clearly } \quad \begin{array}{cc}
\alpha_{1}+\alpha_{2}<\pi, & \alpha_{3}+\alpha_{4}<\pi \\
\alpha_{2}+\alpha_{3} & =\beta_{1}+\beta_{4} .
\end{array}
$$

Together with (3) these imply that

$$
\alpha_{4}+\beta_{4}<\pi
$$

This means that we could use $i=2$ to construct a convex parallel-sided $2 n$-gon $L$ unless

$$
\alpha_{3}+\beta_{3} \geqslant \pi \text {. }
$$

We have shown: if the sum of the angles on the left hand side of $V_{1} V_{n+1}$ is at least $\pi$ then we could use the diagonal $V_{2} V_{n+2}$ unless the sum of the angles on its left hand side is at least $\pi$. This argument can be repeated for each diagonal $V_{i} V_{n+i}$. But if $i=n+1$ the diagonal is $V_{n+1} V_{1}$ and the angles to its left hand side are $\alpha_{2}$ and $\beta_{2}$. By (4) the sum of these is less than $\pi$. This implies that at least one choice of $i$ will allow us to construct a convex parallel-sided $2 n$-gon with area $a(2 n)$. 
Now suppose that a parallel-sided $2 n$-gon has vertices on the lattice points $\left(X_{1}, Y_{1}\right),\left(X_{2}, Y_{2}\right), \ldots,\left(X_{2 n}, Y_{2 n}\right)$, ordered in a clockwise direction. We form a set of $2 n$ vectors $v_{1}, v_{2}, \ldots, v_{2 n}$ by taking the differences between consecutive vertices:

$$
\begin{aligned}
\mathbf{v}_{i} & =\left(X_{i+1}-X_{i}, Y_{i+1}-Y_{i}\right) \text { for } i=1, \ldots, 2 n-1, \\
\mathbf{v}_{2 n} & =\left(X_{1}-X_{2 n}, Y_{1}-Y_{2 n}\right) .
\end{aligned}
$$

Since the $2 n$-gon is parallel-sided we have

$$
\mathbf{v}_{i+n}=-\mathbf{v}_{i} \text { for } i=1, \ldots, n,
$$

so that the sequence $v_{1}, \ldots, v_{n}$ uniquely determines the $2 n$-gon. It is clear that for $n$ of the vectors the $x$-component is non-negative and for the rest it is non-positive. Without loss of generality we may assume that $v_{1}$ to $v_{n}$ have non-negative $x$-components. We call the sequence $\left\{v_{1}, \ldots, v_{n}\right\}$ the edge vectors of the polygon. We denote the components of $v_{i}$ by $x_{i}$ and $y_{i}$ with $x_{i} \geqslant 0$.

THEOREM 3. A necessary and sufficient condition for convexity of a parallel-sided $2 n$-gon with edge vectors $\left\{v_{1}, \ldots, v_{n}\right\}$ is that for $n+1 \geqslant j>i \geqslant 1$

$$
y_{i} x_{j}-x_{i} y_{j}>0
$$

with $\left(x_{n+1}, y_{n+1}\right)$ being interpreted as $\left(-x_{1},-y_{1}\right)$ in accordance with (5).

Proof: A necessary and sufficient condition for convexity is that the angle between $v_{i}$ and $v_{j}$, measured clockwise from $v_{i}$, is less than $\pi$ radians. Let this angle be $\theta$. The expression in (6) gives the cross-product $\mathbf{v}_{j} \times v_{i}$ which is pointing in the $z$ direction if (6) holds. The sign of the left hand side of (6) is the sign of the sine of $\theta$. So (6) holds if and only if $0<\theta<\pi$, as required.

Using edge vectors to define a parallel-sided $2 n$-gon allows us a convenient formula for its area.

THEOREM 4. The area of a parallel-sided convex $2 n$-gon with edge vectors $\left\{\left(x_{i}, y_{i}\right): i=1, \ldots, n\right\}$ is

$$
\sum_{i=1}^{n-1} \sum_{j=i+1}^{n}\left(y_{i} x_{j}-x_{i} y_{j}\right) .
$$

Proof: The summand in ( 7$)$ is the area of a parallelogram with vertices at $(0,0)$, $\left(x_{i}, y_{i}\right),\left(x_{j}, y_{j}\right)$ and $\left(x_{i}+x_{j}, y_{i}+y_{j}\right)$. A convex parallel-sided $2 n$-gon can be partitioned into $\left(\begin{array}{l}n \\ 2\end{array}\right)$ parallelograms each of which has sides equalling a different pair of edge vectors. The reader may be convinced of this by considering Diagram 3. Then (7) is simply the sum of the areas of these $\left(\begin{array}{l}n \\ 2\end{array}\right)$ parallelograms.

We obtain two corollaries to this theorem. 


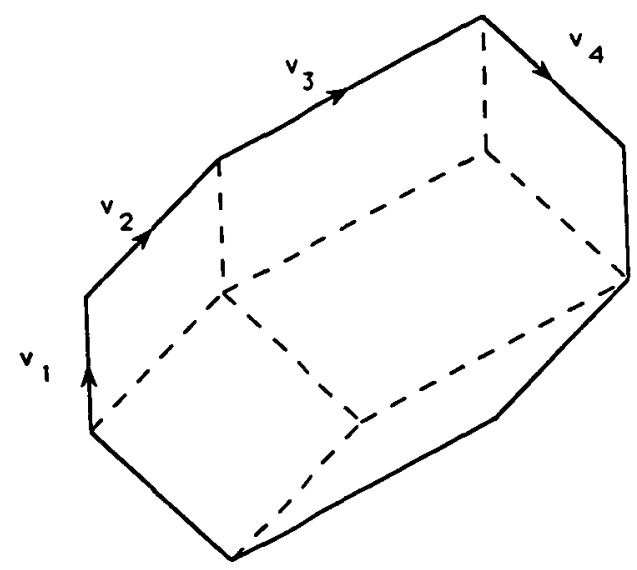

Diagram 3

COROLlary 5. For all $n$ greater than 1 ,

$$
a(2 n) \geqslant\left(\begin{array}{l}
n \\
2
\end{array}\right) .
$$

Proof: Each of the $\left(\begin{array}{l}n \\ 2\end{array}\right)$ summands in (7) is at least 1 .

COROLLARY 6 . If $\left(x_{i}, y_{i}\right)$ is an edge vector of a minimal parallel-sided $2 n$-gon, then $\operatorname{gcd}\left(x_{i}, y_{i}\right)=1$.

Proof: If the components $x_{i}$ and $y_{i}$ of an edge vector have greatest common divisor $d>1$, then we can replace the vector with $\left(x_{i} / d, y_{i} / d\right)$. It is clear from Theorems 3 and 4 that the polygon thus obtained will be convex and have a reduced area.

Thus when seeking $2 n$-gons of minimal area we need only consider polygons with edge vectors whose components are relatively prime. Geometrically this means that the only lattice points on the circumference are the vertices, the necessity of which was noted in the proof of Theorem 1.

For the final results in this section we need the following theorem which concerns the effect of a unimodular transformation on a parallel-sided convex $2 n$-gon.

THEOREM 7. If $\left\{\left(x_{i}, y_{i}\right): i=1, \ldots, n\right\}$ is the sequence of edge vectors of a parallel-sided convex $2 n$-gon, and $M$ is a $2 \times 2$ matrix with integer entries and determinant 1 , then

$$
\left\{\left(x_{i}, y_{i}\right) M: i=1, \ldots, n\right\}
$$

is the sequence of edge vectors of another parallel-sided convex $2 n$-gon with the same area. 
Proof: Suppose

$$
M=\left[\begin{array}{ll}
a & b \\
c & d
\end{array}\right]
$$

Then for any $i$ let

$$
\begin{aligned}
\left(x_{i}^{\prime}, y_{i}^{\prime}\right) & =\left(x_{i}, y_{i}\right) M \\
& =\left(a x_{i}+c y_{i}, b x_{i}+d y_{i}\right) .
\end{aligned}
$$

It is easily checked that

$$
y_{i}^{\prime} x_{j}^{\prime}-x_{i}^{\prime} y_{j}^{\prime}=y_{i} x_{j}-x_{i} y_{j}
$$

Theorem 3 then implies that the new polygon is convex and Theorem 4 implies that the two polygons have equal area.

COROLLARY 8. There exists a parallel-sided minimal $2 n$-gon with edge vectors $\left\{\left(x_{i}, y_{i}\right): i=1, \ldots, n\right\}$ satisfying

$$
\left(x_{1}, y_{1}\right)=(0,1)
$$

and

$$
y_{i} \geqslant x_{i}>0 \text { for } i=2, \ldots, n .
$$

Proof: In the remarks preceding Theorem 3 we showed that such a polygon exists with each $x_{i} \geqslant 0$. By Corollary $6 \operatorname{gcd}\left(x_{1}, y_{1}\right)=1$ so there exist integers $b$ and $d$ such that

$$
b x_{1}+d y_{1}=1 .
$$

We now post-multiply each edge vector by the matrix

$$
M=\left[\begin{array}{cc}
y_{1} & b \\
-x_{1} & d
\end{array}\right]
$$

This matrix has determinant 1 so we may apply Theorem 7 , and obtain the sequence of edge vectors of a new minimal $2 n$-gon with edge vectors $\left\{\left(x_{i}^{\prime}, y_{i}^{\prime}\right): i=\right.$ $1, \ldots, n\}$. It is easily checked that

$$
\left(x_{1}^{\prime}, y_{1}^{\prime}\right)=(0,1)
$$

and that for $n \geqslant i>1$,

$$
x_{i}^{\prime}=y_{1} x_{i}-x_{1} y_{i}
$$

which is positive by Theorem 3. Thus we have a minimal $2 n$-gon satisfying ( 8 ) and with $x$-components of all but the first edge vector strictly positive. 
Now post-multiply each new edge vector by the matrix

$$
\left[\begin{array}{cc}
1 & N \\
0 & 1
\end{array}\right]
$$

where $N$ is a positive integer.

This does not change the $x$-components of the vectors, nor does it change $\left(x_{1}^{\prime}, y_{1}^{\prime}\right)$. However it does increase the $y$ components of the other edge vectors. If the integer $N$ is sufficiently large we obtain a vector sequence with $y_{i} \geqslant x_{i}$ for each $i$, as required. [

We now summarise the conclusions of this section. We have

$$
a(2 n)=\min \left\{\sum_{i=1}^{n-1} \sum_{j=i+1}^{n}\left(y_{i} x_{j}-x_{i} y_{j}\right)\right\}
$$

where the minimum is taken over all sequences of $n$ ordered pairs

$$
\left\{\left(x_{i}, y_{i}\right): i=1, \ldots, n\right\}
$$

satisfying,

$$
\begin{array}{lll}
y_{i} x_{j}-x_{i} y_{j}>0 & \text { for } & 1 \leqslant i<j \leqslant n, \\
\operatorname{gcd}\left(x_{i}, y_{i}\right)=1 & \text { for } & i=1, \ldots, n, \\
\left(x_{1}, y_{1}\right)=(0,1), & \\
y_{i} \geqslant x_{i}>0 & \text { for } & i=2, \ldots, n .
\end{array}
$$

\section{FAREy sequences of vectors.}

We define a sequence of Farey sequences of vectors $S_{0}, S_{1}, \ldots$ by

and if

$$
S_{0}=\{(0,1),(1,1)\}
$$

then

$$
S_{i}=\left\{u_{1}, u_{2}, \ldots, u_{m}\right\}
$$

Thus

$$
\begin{gathered}
S_{i+1}=\left\{\mathbf{u}_{1}, \mathbf{u}_{1}+\mathbf{u}_{2}, \mathbf{u}_{2}, \mathbf{u}_{2}+\mathbf{u}_{3}, \ldots, \mathbf{u}_{m}\right\} . \\
S_{1}=\{(0,1),(1,2),(1,1)\}, \\
S_{2}=\{(0,1),(1,3),(1,2),(2,3),(1,1)\},
\end{gathered}
$$

and so on. The construction of these sequences is illustrated by the digraph in Diagram 4. These sequences are analogous to the well-known Farey sequences of fractions (see, for instance, [3]) in which our $x$ and $y$ components become numerators and denominators 


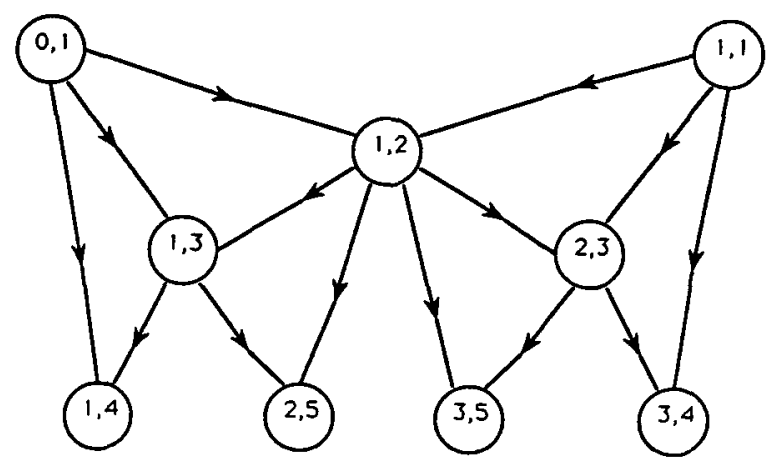

Diagram 4

of rational numbers. The following properties of our vector sequences are easily proven and are analogous to properties of the fraction sequences.

(a) If $j>i$ and $\mathbf{u}_{j}=\left(s_{j}, t_{j}\right), \mathbf{u}_{i}=\left(s_{i}, t_{i}\right)$ are members of $S_{k}$ for some $k$ then

$$
t_{i} s_{j}-s_{i} t_{j}>0 \text {. }
$$

(b) If $\operatorname{gcd}(x, y)=1$ and $y \geqslant x \geqslant 0$ then $(x, y)$ belongs to $S_{k}$ for some $k$.

(c) $(0,1) \in S_{k}$ for all $k$.

Comparing these properties with (10), (11), (12), and (13), we see that a minimal parallel-sided $2 n$-gon can be constructed with its edge vector sequence being a subsequence of some Farey sequence of vectors.

Before proving Theorem 9 , which is the major result of this paper, we need some more notation.

We write the vectors in the $k$ th Farey vector sequence as

$$
\mathbf{u}(k, 1), \mathbf{u}(k, 2), \ldots, \mathbf{u}(k, m) .
$$

If $\mathbf{v}=(x, y)$ and $\mathbf{u}=(s, t)$ we write

$$
\mathbf{u} \gtrsim \mathbf{v}
$$

to mean

$$
y s-x t>0 \text {. }
$$

When $x$ and $s$ are positive this is equivalent to

$$
\frac{y}{x}>\frac{t}{s}
$$

that is, the slope of $\mathbf{v}$ is greater than the slope of $\mathbf{u}$. It follows from property (a) above that if $j>i$ than $u(k, j) \gtrsim u(k, i)$, and from (10) that $v_{j} \gtrsim v_{i}$ for edge vectors $v_{i}$ and $\mathbf{v}_{j}$. 
THEOREM 9. Suppose that $K$ is a minimal parallel-sided $2 n$-gon with edge vectors $\left\{\mathbf{v}_{1}, \mathbf{v}_{2}, \ldots, \mathbf{v}_{n}\right\}=\left\{\left(x_{i}, y_{i}\right): i=1, \ldots, n\right\}$ which satisfy (10), (11), (12) and (13).

If

$$
\mathbf{v}_{i}=\mathbf{u}(k, j)
$$

in $S_{k}$ for some $k \geqslant 1$ and $\mathbf{v}_{i} \notin S_{k-1}$, then, without loss of generality, $u(k, j-1)$ and $\mathrm{u}(k, j+1)$ belong to the sequence of edge vectors.

Proof: Suppose

$$
\begin{aligned}
\mathbf{v}_{i} & =\mathbf{u}(k, j) \\
& =\mathbf{u}(k, j-1)+\mathbf{u}(k, j+1)
\end{aligned}
$$

for some $j$ and $k$. This implies that $\mathbf{v}_{i} \in S_{k}$ and $\mathbf{v}_{i} \notin S_{k-1}$. Suppose that $\mathrm{u}(k, j-1)$ is not an edge vector and further suppose that $k$ is the greatest index for which such an $i$ can be found.

This last assumption implies that

$$
\mathbf{v}_{i-1} \lesssim \mathrm{u}(k, j-1)
$$

for $v_{i-1} \gtrsim u(k, j-1)$ would contradict the maximality of $k$. This fact will be apparent from the digraph in Diagram 4.

Now let $r$ be the greatest integer satisfying

$$
\mathbf{v}_{i+r} \lesssim \mathbf{u}(k, j+1), \mathbf{v}_{i+r} \neq \mathrm{u}(k, j+1)
$$

Clearly (15) holds with $r=0$ so $r$ is non-negative.

The maximality of $k$ now implies that

$$
\begin{aligned}
\mathbf{v}_{i+1} & =\mathbf{v}_{i}+\mathbf{u}(k, j+1) \\
\mathbf{v}_{i+2} & =\mathbf{v}_{i+1}+\mathbf{u}(k, j+1), \\
\vdots & \\
\mathbf{v}_{i+r} & =\mathbf{v}_{i+r-1}+u(k, j+1) .
\end{aligned}
$$

If any of these did not hold, then the right hand side would give us a $u\left(k^{\prime}, j-1\right)$ with $k^{\prime}>k$. Hence, by (14)

$$
\mathbf{v}=\mathbf{u}(k, j-1)+(s-i+1) \mathrm{u}(k, j+1), \quad s=i, \ldots, i+r
$$


We now show that we can obtain a parallel-sided convex polygon $K^{\prime}$ whose area is not more than the area of $K$. The edge vectors of $K^{\prime}$ are $\mathbf{v}_{1}^{\prime}, \mathbf{v}_{2}^{\prime}, \ldots, \mathbf{v}_{n}^{\prime}$ where

$$
\begin{aligned}
\mathbf{v}_{m}^{\prime} & =\mathbf{v}_{m}, \quad \text { for } \quad m=1, \ldots, i-1, \\
\mathbf{v}_{m}^{\prime} & =\mathbf{u}(k, j-1)+(m-i) \mathbf{u}(k, j+1), \\
& =\mathbf{v}_{m}-\mathbf{u}(k, j+1) \text { for } \quad m=i, \ldots, i+r \\
\mathbf{v}_{m}^{\prime} & =\mathbf{v}_{m}, \quad \text { for } \quad m=i+r+1, \ldots, n
\end{aligned}
$$

To show that $K^{\prime}$ is convex we need to establish that

$$
\mathbf{v}_{m}^{\prime} \leq \mathbf{v}_{m+1}^{\prime}
$$

for $m=1, \ldots, n-1$. This is clear for all $m$ except $m=i-1$ and this case was established in (15). It remains to show that its area is not greater than the area of $K$.

Writing $A$ for the area of $K$ and $A^{\prime}$ for the area of $K^{\prime}$ we have

$$
\begin{aligned}
A & =\sum_{i=1}^{n-1} \sum_{j=i+1}^{n} \mathbf{v}_{j} \times \mathbf{v}_{i} \\
& =S_{1}+S_{2}+\ldots+S_{6}
\end{aligned}
$$

where

$$
S_{1}=\sum_{i=1}^{i-2} \sum_{t=t+1}^{i-1} v_{t} \times v_{t}
$$

$$
\begin{aligned}
& S_{2}=\sum_{s=1}^{i-1} \sum_{t=i}^{1+r} v_{t} \times v_{s} \\
& S_{3}=\sum_{i=1}^{i-1} \sum_{t=i+1}^{n} v_{t} \times v_{s} \\
& S_{4}=\sum_{s=i}^{i+r-1} \sum_{t=s+1}^{i+r} v_{t} \times v_{t} \\
& S_{5}=\sum_{s=i}^{i+r-1} \sum_{t=i+r+1}^{n} v_{t} \times v_{s} \\
& S_{6}=\sum_{\bullet=i+r+1}^{n-1} \sum_{t=s+1}^{n} v_{t} \times v_{\bullet} .
\end{aligned}
$$

We write $S_{j}^{\prime}$ for the sums corresponding to $S_{j}, j=1, \ldots, 6$, but with summand $v_{t}^{\prime} \times v^{\prime}$, instead of $v_{t} \times v_{,}$. From (17) we get, writing $u^{+}$for $u(k, j+1)$ and $u^{-}$for 
$\mathbf{u}(k, j-1)$

$$
\begin{aligned}
& S_{1}^{\prime}=S_{1}, \\
& S_{2}^{\prime}=S_{2}-\sum_{i=1}^{i-1} \sum_{t=i}^{i+r}\left(u^{+} \times v_{s}\right) \\
& =S_{2}-(r+1) \sum_{\imath=1}^{i-1}\left(\mathbf{u}^{+} \times v_{\imath}\right) \text {, } \\
& S_{3}^{\prime}=S_{3} \text {, } \\
& S_{4}^{\prime}=\sum_{s=i}^{i+r-1} \sum_{t=s+1}^{i+r}\left(v_{t}-u^{+}\right) \times\left(v_{e}-u^{+}\right) \\
& =\sum_{\imath=i}^{i+r-1} \sum_{t=\ell+1}^{i+r}\left\{v_{t} \times v_{\varepsilon}-v_{t} \times u^{+}-\mathbf{u} \times v_{\imath}+u^{+} \times u^{+}\right\}
\end{aligned}
$$

Now $\mathbf{u}^{+} \times \mathbf{u}^{+}=0$. Using (17) we then get

$$
\begin{aligned}
S_{4}^{\prime} & =S_{4}-\sum_{i=i}^{i+r-1} \sum_{t=s+1}^{i+r}\left\{\left\{\left(u^{-}+(t-i+1) u^{+}\right) \times u^{+}\right\}\right. \\
& \left.+\left\{u^{+} \times\left(u^{-}+(s-i+1) u^{+}\right)\right\}\right\} \\
= & S_{4}-\sum_{i=i}^{i+r-1} \sum_{t=t+1}^{i+r}\left(u^{-} \times u^{+}+u^{+} \times u^{-}\right) \\
& =S_{4}, \\
S_{5}^{\prime} & =S_{5}-(r+1) \sum_{t=i+r+1}^{n}\left(v_{t} \times u^{+}\right), \\
S_{6}^{\prime} & =S_{6} .
\end{aligned}
$$

Noting that $A^{\prime}=S_{1}^{\prime}+S_{2}^{\prime}+\ldots+S_{6}^{\prime}$ we obtain

$$
A^{\prime}=A-(r+1)\left\{\sum_{s=1}^{i-1}\left(\mathbf{u}^{+} \times \mathrm{v}_{0}\right)+\sum_{t=i+r+1}^{n}\left(\mathrm{v}_{t} \times \mathbf{u}^{+}\right)\right\}
$$

Now (15) implies that $\mathbf{u}^{+} \gtrsim \mathbf{u}^{-} \gtrsim v$, for $s=1, \ldots, i-1$ and (16) implies that $\mathbf{v}_{t}=\mathbf{u}^{+}$or $\mathbf{v}_{t} \gtrsim \mathbf{u}^{+}$for $t=i+r+1, \ldots, n$. Thus each summand is non-negative. We then have

$$
A^{\prime} \leqslant A
$$

as required. 
A similar analysis applies when $\mathrm{u}(k, j+1)$ rather than $\mathrm{u}(k, j-1)$ is absent from the set of edge vectors.

In graph-theoretic terms this theorem says that without loss of generality the sequence of edge vectors of a minimal $2 n$-gon is a closure of the digraph in Diagram 4; that is, a set of vertices with the property that the end-points of the out-arcs from any vertex in the set are also in the set.

For any $n$ there is a finite number of closures of $n$ vertices. We can calculate the areas corresponding to each such closure, and the minimum of these equals $a(2 n)$.

The number of such closures increases quickly with $n$. For $n=2$ there is only one choice: $\{(0,1),(1,1)\}$. Similarly for $n=3:\{(0,1),(1,2),(1,1)\}$. For $n=4$ there are two choices and for $n=5$ there are five. Values of $a(2 n)$ for $n=2, \ldots, 11$ are shown in Table 2. For each $n$ it is possible to find a minimal $2 n$-gon using the edge vectors of a minimal $2(n-1)$-gon with an extra edge. We start with edge $(0,1)$ then add the new edges to the edge sequence.

\begin{tabular}{rcc}
$\mathbf{n}$ & $a(2 n)$ & New edge \\
\hline 2 & 1 & $(1,1)$ \\
3 & 3 & $(1,2)$ \\
4 & 7 & $(1,3)$ \\
5 & 14 & $(1,4)$ \\
6 & 24 & $(2,5)$ \\
7 & 40 & $(1,5)$ \\
8 & 59 & $(2,7)$ \\
9 & 87 & $(1,6)$ \\
10 & 121 & $(2,9)$ \\
11 & 164 & $(1,7)$ \\
\hline
\end{tabular}

Table 2: Values for $a(2 n)$. The values for $n=6, \ldots, 11$ are original to this paper.

$V$-GONS WITH $v$ ODD.

Our results so far have been concerned with $v$-gons in which $v$ is even. In the next theorem we obtain bounds on $a(2 n+1)$.

THEOREM 10. For $n \geqslant 2$ we have

(a) $a(2 n+1) \geqslant[(a(2 n+2)+a(2 n)) / 2]+1 / 2$,

(b) $a(2 n+1) \leqslant a(2 n+2)-1 / 2$,

where square brackets denote integer part. 
Proof: (a) Suppose $K$ is a minimal $(2 n+1)$-gon. By drawing a line from vertex $i$ to vertex $i+n$ we partition $K$ into two areas $A_{1}$ and $A_{2}$. By an argument similar to that used in the proof of Theorem 2, $i$ can be chosen so that each area is half a convex polygon. Then one of these areas is at least $a(2 n) / 2$ and the other is at least $a(2 n+2) / 2$. By the remark following Theorem $1, a(2 n+1)$ cannot be an integer and (a) follows.

(b) Now suppose $K$ is a minimal $(2 n+2)$-gon. If we remove vertex $i$ and form an edge from vertex $i-1$ to vertex $i+1$, we form a convex $(2 n+1)$-gon. The triangle thus removed has area at least half by Pick's Theorem and (b) follows.

Corollary 11.

(a) $19.5 \leqslant a(11) \leqslant 21.5$,

(b) $a(13)=32.5$,

(c) $49.5 \leqslant a(15) \leqslant 54.5$,

(d) $73.5 \leqslant a(17) \leqslant 86.5$,

(e) $104.5 \leqslant a(19) \leqslant 120.5$,

(f) $142.5 \leqslant a(21) \leqslant 163.5$.

Proof: These results follow from the theorem and the data in Tables 1 and 2. [

In Corollary 5 we showed that

$$
a(2 n) \geqslant\left(\begin{array}{l}
n \\
2
\end{array}\right)
$$

In [5] Rabinowitz showed that

$$
g(2 n) \leqslant\left(\begin{array}{l}
n \\
3
\end{array}\right)
$$

By Theorem 1 this implies:

$$
a(2 n) \leqslant\left(\begin{array}{l}
n \\
3
\end{array}\right)+n-1 .
$$

This result can be obtained by calculating the area of a $2 n$-gon with edge vectors $(0,1),(1, n-1),(1, n-2), \ldots,(1,1)$. This bound is only sharp for $n \leqslant 5$, while (21) is only sharp for $n \leqslant 3$.

\section{REFERENCES}

[1] J.R. Arkinstall, 'Minimal requirements for Minkowski's theorem in the plane I', Bull. Austral. Math. Soc. 22 (1980), 259-274. 
[2] H.S.M. Coxeter, Introduction to Geometry (John Wiley and Sons, Inc, New York, 1980).

[3] G.H. Hardy and E.M. Wright, An Introduction to the Theory of Numbers, Fourth Edition (Oxford, 1975).

[4] Stanley Rabinowitz, Convex Lattice Polygons, Ph.D. Dissertation (Polytechnic University, Brooklyn, New York, 1986).

[5] Stanley Rabinowitz, 'On the number of lattice points inside a convex lattice n-gon', Congr. Numer. 73 (1990), 99-124.

School of Mathematics and Statistics

Curtin University of Technology

GPO Box U 1987

Perth WA 6001

Australia 